\title{
Measuring Cell Growth and Junction Development in Epithelial Cells Using Electric Cell- Substrate Impedance Sensing (ECIS) \\ Shaista Anwer ${ }^{1}$ and Katalin Szaszi ${ }^{1,2, *}$
}

\author{
${ }^{1}$ Keenan Research Centre for Biomedical Science of the St. Michael's Hospital, Toronto, Canada; ${ }^{2}$ Dept. \\ of Surgery, University of Toronto, ON, Canada \\ *For correspondence: katalin.szaszi@unityhealth.to
}

\begin{abstract}
[Abstract] Electric Cell-substrate Impedance Sensing (ECIS) is an automated method that can be used to quantify processes such as cell attachment, growth, migration and barrier functions (i.e., the properties of tight junctions). The method provides simultaneous information on cell number and tight junction function by detecting electric parameters of cells grown on electrodes. Samples are probed with small alternating current $(\mathrm{AC})$ over a range of frequencies, and changes in capacitance and impedance are measured over time. Capacitance reflects the degree of electrode coverage by cells, that correlates with cell number, and can be used to assess cell proliferation or migration. Impedance values inform about barrier function. Obtaining real-time simultaneous information on these parameters is unique to this system and is of great value for addressing fundamental questions such as the role of tight junction proteins in cell growth and migration. This protocol describes the use of ECIS to follow cell growth and tight junction-dependent barrier generation in tubular epithelial cells. We used this method to explore how depleting claudin-2, a tight junction protein affects tubular cell growth and barrier function. During the process, cells are transfected with control or claudin-2-specific siRNA, and 24h later plated on electrodes. ECIS automatically collects information on cell growth and barrier as the monolayer develops. The data are initially analyzed using the ECIS software and exported into a graph software for further processing.
\end{abstract}

Keywords: Impedance sensing, Epithelial cells, Cell growth, Transepithelial resistance, Tight junction development

[Background] Epithelial cells generate monolayers characterized by well-developed intercellular junctions. Input from the junctions determines cell behavior, including proliferation and migration. As junctions develop, cell proliferation and migration slows and then halts due to contact inhibition (McClatchey and Yap, 2012). Although the past decades rapidly augmented our understanding of the molecular mechanisms of proliferation and migration, many aspects of these processes, and the exact nature of contact inhibition remain incompletely defined. As cells establish a confluent layer, intercellular junctions, including tight junctions develop and mature, ensuring well-controlled paracellular permeability (barrier functions) (Zihni et al., 2016). Paracellular seal and permeability pathways are established by the claudin family of tight junction proteins (Tsukita et al., 2019). Interestingly, accumulating evidence suggests that in addition to their role as permeability regulators, many claudins also modulate proliferation and migration (Hagen, 2017; Venugopal et al., 2019). These roles, however, 
remain incompletely understood. Methods that follow barrier function and cell growth or migration simultaneously over time are crucial for obtaining new insights. Electric Cell-substrate Impedance Sensing (ECIS), that was developed by Applied BioPhysics offers this possibility. ECIS is an impedancebased, automated method that allows the quantification of various aspects of cell behavior through detection of electric properties (Wegener et al., 2000). The cells are grown in chambers containing small gold electrodes (referred to as arrays), and the system continuously probes the electrodes using small, biologically inert alternating current. A range of $A C$ frequencies from $62.5-64,000 \mathrm{~Hz}$ is used to measure impedance and capacitance across the electrodes over time. The basis of the measurement is that the current can flow through the cells or under and between them, and the magnitude of resistance of these compartments depends on the frequency of the AC current applied. Thus, specific frequencies can be used to probe different parameters, and this can be utilized to follow various aspects of cell behavior in the same sample (Stolwijk et al., 2015; Wegener et al., 2000). During measurment the system measures capacitance and impedance at the full frequency range, and the user can select the required parameters during analysis. Capacitance detected using high $\mathrm{AC}$ frequency reflects coverage of the electrode, with little influence from other parameters. Capacitance data collected in a typical experiment are shown in Figure $1 \mathrm{~A}$ (top graph). This demonstrates that following seeding cells generate an insulating layer on the electrodes that is reflected by a drop in capacitance. Capacitance reaches its minimum and stabilizes when a fully confluent layer is generated. The initial rate of drop reflects cell attachment, that is completed by $3 \mathrm{~h}$. After this time, the rate of drop reflects cell growth. Figure $1 \mathrm{~A}$ bottom graph shows the parallel changes in impedance $(Z)$ values measured at low $A C$ frequencies. In epithelial cells with welldeveloped tight junctions, impedance measured at $500 \mathrm{~Hz}$ or lower frequencies reflects predominantly the properties of the paracellular space. Impedance increases as cells reach confluence and tight junctions develop. Many epithelial cells show an increase in the $Z$ value, followed by a decrease as junctions mature (Amoozadeh et al., 2017). Interestingly, simultaneous measurement of capacitance and impedance reveals that tight junction development and maturation does not stop as confluence is reached (Amoozadeh et al., 2018). More detailed analysis to separate different components of impedance can be performed using mathematical modelling, built into the software, which can be advantageous in cells with weaker paracellular resistance (e.g., endothelial cells) (Wegener et al., 2000; Stolwijk et al., 2015; García et al., 2019). Although the theory of mathematical modelling in ECIS is complex, the measurement itself is relatively straight forward, and simple analysis can provide valuable information. ECIS is also equipped with an elevated field module for wounding of the layer, and capacitance values can be used for an automated cell migration assay (not discussed in this protocol) (Szaszi et al., 2012).

The advantages of ECIS are as follows. It is an automated, continuous, real-time, label-free detection method. Since the arrays are housed in a tissue culture incubator, the environment is controlled and any measurement artifacts stemming from changes in temperature and $\mathrm{CO}_{2}$ levels are eliminated. In comparison with other methods that follow cell growth or migration, the key benefit of ECIS is the ability to simultaneously detect transepithelial resistance (TER), i.e., barrier function and cell growth or migration (Kakiashvili et al., 2011; Amoozadeh et al., 2015 and 2017; Stolwijk et al., 2015; Láng et al., 
2017) (Figure 1). This can be valuable for correlating effects of various treatments on these highly interdependent parameters, thereby providing new insights into fundamental cell biological questions.

This protocol describes the use of ECIS to follow tubular epithelial cell growth, and the development of barrier via formation of the tight junctions. We used this protocol to assess the development of cell confluence (Amoozadeh et al., 2018) and to follow effects of silencing of a tight junction protein, claudin2 rate on proliferation rate and barrier function (Dan et al., 2019). Silencing claudin-2 alters both barrier function (resulting in elevated resistance) (Amoozadeh et al., 2018) and cell growth (reducing proliferation) (Dan et al., 2019). Simultaneous detection of these parameters reveals both effects. We found that claudin-2 silencing slows the development of confluency, but does not prevent it, and claudin2 depleted cells generate a barrier which is tighter that the control due to absence of the channel forming claudin-2. Adaptation of the method for other cell types and proteins requires optimization of the seeding conditions, electrode coating and transfection parameters. We provide notes on these issues to direct the reader.

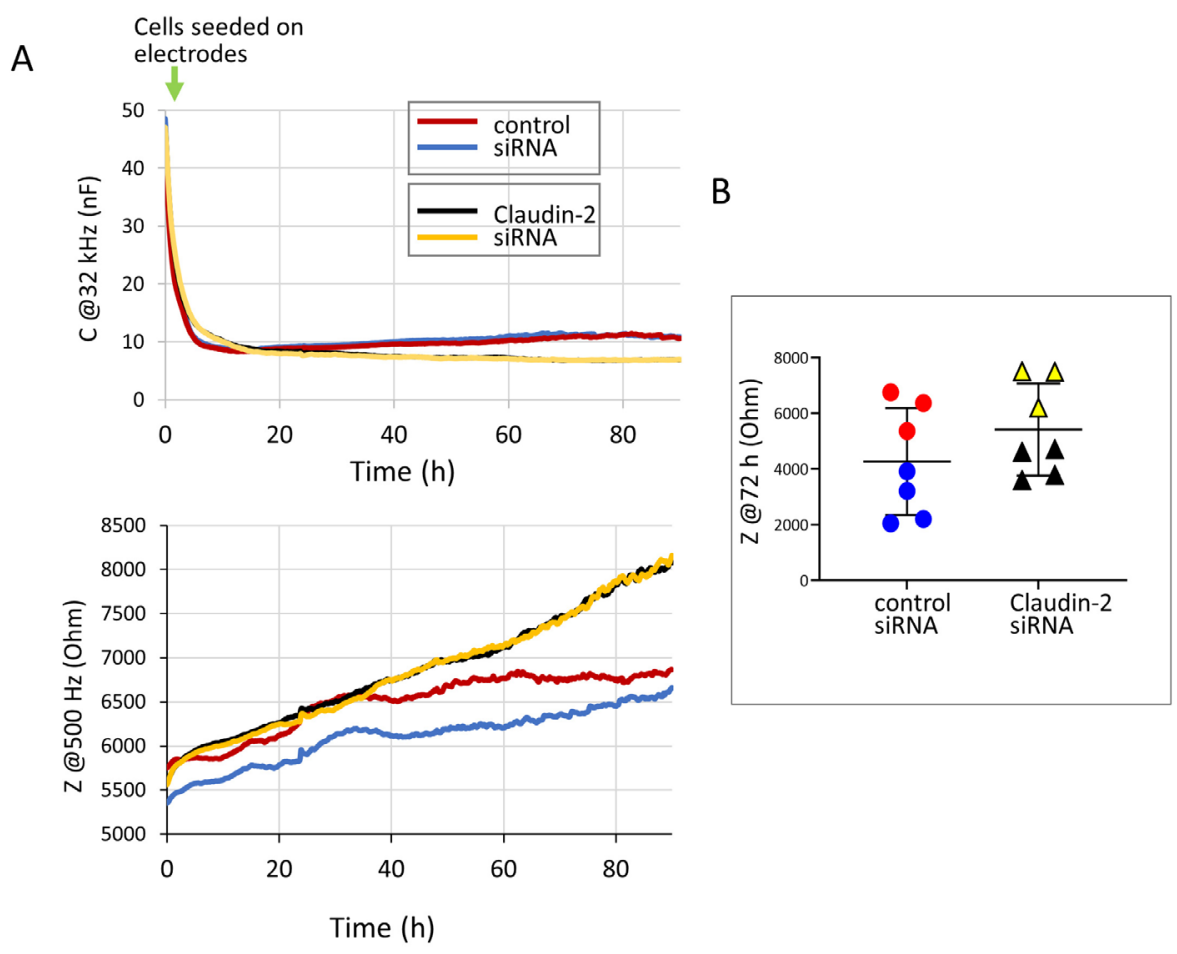

Figure 1. Capacitance and impedance measurements of LLC-PK1 cells with or without claudin-2 silencing. A. Exported graphs showing simultanously detected changes in capacitance at $32 \mathrm{kHz}$ (reflecting cell growth) and impedance (z) at $500 \mathrm{kHz}$ (reflectig barrier). LLC-PK1 cells were transfected using control or claudin-2-specific siRNA and electrical properties were measured using ECIS, as described in the protocol. B. Impedance ( $\mathrm{z}$ ) was determied at $72 \mathrm{~h}$ after seeding of cells in two different experiments. The data from the same experiment are indicated by black and red for experiment \#1 and blue and green for experiment \#2 (control and claudin-2 siRNA, respectively). 


\section{Materials and Reagents}

1. Clear tissue culture-treated 6-well microplates (Corning Costar ${ }^{\circledR}$, Millipore-Sigma, catalog number: CLS3513)

2. Microcentrifuge tubes ( $1.5 \mathrm{ml})$ (Fisherbrand, catalog number: 2043-05408129)

3. Countess ${ }^{\mathrm{TM}}$ Cell Counting Chamber Slides (Thermo Fisher, Invitrogen, catalog number: C10228)

4. Cell line: LLC-PK ${ }_{1}$ kidney tubule epithelial cell line (European Collection of Authenticated Cell Cultures, catalog number: 86121112)

5. Cell culture media and reagents:

Dulbecco's Modified Eagle Medium (D-MEM), low glucose, with L-glutamine and $110 \mathrm{mg} / \mathrm{L}$ sodium pyruvate (Gibco, Thermo Fisher, catalog number: 11885084)

Fetal Bovine Serum (Gibco, Thermo Fisher, catalog number: 12483-020)

Penicillin-Streptomycin, 100x, sterile-filtered, cell culture tested (Sigma-Aldrich, catalog number: P-4333. 25300062)

Trypsin-EDTA (0.05\% Trypsin with EDTA 4Na) (Thermo Fisher, Gibco, catalog number 25300062)

Opti-MEM ${ }^{\top M}$ I Reduced Serum Medium for transfection (Thermo Fisher, Gibco, catalog number: 31985088)

6. Trypan Blue Stain (0.4\%) (Gibco, catalog number: 15250061) for use with the Countess ${ }^{\mathrm{TM}}$ Automated Cell Counter (Invitrogen, Thermo Fisher, catalog number T10282)

7. Lipofectamine ${ }^{T M}$ RNAiMAX Transfection Reagent (Invitrogen, Thermo Fisher, catalog number 13778150)

8. Two custom-designed siRNAs targeting porcine claudin-2 (Thermo Fisher/Dharmacon) (sequence \#1, CCAGAACTCTCGCGCCAAA, and \#2 CCCTGATAGCTGGGATCAT)

9. Silencer ${ }^{\mathrm{TM}}$ Negative Control No. 1 siRNA (non-related (NR)) siRNA (Applied Biosystems/Ambion, Thermo Fisher, catalog number: AM4611)

10. ECIS electrode array, type: $8 \mathrm{~W} 10 \mathrm{E}$ or $8 \mathrm{~W} 10 \mathrm{E}+\mathrm{PET}$ (Applied Biophysics, catalog number: $8 \mathrm{~W} 10 \mathrm{E}$ or $8 \mathrm{~W} 10 \mathrm{E}+)$

11. L-cysteine (Millipore-Sigma-Aldrich catalog number: C7352)

12. $10 \mathrm{mM} \mathrm{L-cysteine} \mathrm{solution} \mathrm{for} \mathrm{electrode} \mathrm{stabilization} \mathrm{(see} \mathrm{Recipes)}$

\section{Equipment}

1. $\mathrm{ECIS}^{\circledR}$ Z-Theta instrument with 16-well array module housed in incubator (Applied Biophysics)

2. Countess ${ }^{\mathrm{TM}}$ Automated Cell Counter (Thermo Fisher, Invitrogen, catalog number C10228)

3. Tissue culture equipment (sterile hood, incubator, centrifuge) 


\section{Software}

1. ECIS Z $\theta$ software

2. Graph software (e.g., Excel or GraphPad)

\section{Procedure}

A. Electrode stabilization with cysteine pretreatment

1. Pretreat electrodes with $200 \mu \mathrm{l}$ of filtered $10 \mathrm{mM}$ L-cysteine for $15 \mathrm{~min}$ at room temperature.

2. Aspirate the cysteine solution and wash with $200 \mu \mathrm{l}$ of autoclaved $\mathrm{dH}_{2} \mathrm{O}$ twice. Do not wash electrodes with PBS.

3. Air dry the electrodes before seeding cells. Alternatively, the treated and dried electrodes can be stored at room temperature till its expiry.

B. Splitting epithelial cells for transfection with siRNA

1. Grow LLC-PK 1 cells in low glucose DMEM supplemented with $10 \%$ FBS and $1 \%$ Pen-Strep in an incubator at $37^{\circ} \mathrm{C}$ with $5 \% \mathrm{CO}_{2}$.

2. Split cells when they are grown to about $90 \%$ confluence.

3. Seed cells for transfection in 6-well plates using standard cell culture procedures.

4. Use $2 \mathrm{ml}$ antibiotic-free, serum-containing DMEM/well to seed cells. Aim at obtaining $30-40 \%$ confluence the next day.

C. Transfecting cells

The following day (16-24 h later) transfect cells with $100 \mathrm{nM}$ of the negative control (non-related, NR) or claudin-2-specific siRNA using the Lipofectamine RNAiMAX transfection reagent as follows:

1. Mix reagents:

Tube A: For each condition (well) to be transfected, prepare siRNA: dilute $2.4 \mu$ of $100 \mathrm{nM}$ stock siRNA duplex in $200 \mu \mathrm{l}$ Opti-MEM medium without serum in a sterile microcentrifuge tube. Mix gently. For more wells, scale up amounts as needed.

Tube B: For each condition (well) prepare Lipofectamine ${ }^{\mathrm{TM}}$ : add $3 \mu$ Lipofectamine ${ }^{\mathrm{TM}}$ RNAiMAX to $200 \mu$ l Opti-MEM medium. Mix gently. For more wells, scale up amounts as needed.

2. Incubate the tubes for 5 min at room temperature.

3. Mix the content of tube A and B to generate the siRNA-Lipofectamine complex. Incubate for 15 $\min$.

4. At the end of the incubation time, add $400 \mu \mathrm{l}$ of the siRNA-Lipofectamine mix to the corresponding well. Gently shake to mix and return to the incubator.

5. Grow cells for $24 \mathrm{~h}$. 
D. Seeding cells on the electrode arrays

1. The next day, prepare the electrode arrays for seeding:

Add $400 \mu \mathrm{l}$ pre-warmed complete culture medium (growth medium with serum and antibiotics) to the wells, and place into the incubator for $15 \mathrm{~min}$ prior to seeding cells. This will coat the surface with serum and warm the array to reduce convection of the medium/cell suspension due to heat differences, that affects even settling of cells on the electrode.

2. Trypsinize cells transfected with control siRNA and claudin-2-specific siRNA. When cells are floating, add warm medium, collect, and spin down cells using $300 \times \mathrm{g}$. Resuspend in fresh medium. Keep warm while performing the cell counting.

3. Count cells using the Countess automated cell counter:

Using a sterile tip remove $10 \mu \mathrm{l}$ of the cell suspension and mix with $10 \mu \mathrm{l}$ of Trypan blue in a microcentrifuge tube. Add to a Countess ${ }^{\mathrm{TM}}$ Cell Counting Chamber Slide and count cells using the automated cell counter to obtain number of viable cells.

Note: Manual counting can be used instead of the Countess.

4. Adjust volume using warm medium to obtain a total of $0.5 \times 10^{5}$ cells/electrode in a final volume of $400 \mu$ l.

5. Remove array from the incubator and aspirate the medium from the wells. Add $400 \mu$ of cell suspension to each well.

6. Insert the electrode into the ECIS array holder in the incubator and screw the holder tight.

E. Software setup and acquiring data

1. Turn on the computer and the ECIS Z $Z$ machine.

2. Open the ECIS software. Click the "Setup" button. The system will check the connection. If the connection is good, the wells show up as green. If the connection is bad (red wells), reinsert the electrode, then click "Check" to confirm connectivity of all electrodes by using the "Check" button.

3. Select the type of electrode. Choose the "Multiple Frequency/Time (MFT)" option (the required frequency can be selected during analysis).

4. Click on "Start". Enter the file name and any comments you want to save. Collect data for 24$48 \mathrm{~h}$.

5. As cells grow, $\mathrm{C}$ at high frequency ( 32 or $64 \mathrm{kHz}$ ) decreases (see Note 6 for comments on the choice of frequency). Confluence is achieved when $\mathrm{C}$ is stable at the minimum. At this point, the measurement can be stopped.

\section{Data analysis}

1. Prepare data for analysis

In the ECIS software on the right top click the "Analyze" tab. Figure 2 shows a screenshot of the software with a typical measurement, with the corresponding commands labelled. You can select the parameters and curves you want to export. This limits the amount of data exported. 
Alternatively, you can export all data (see below) and process them in the graph software. However, selecting the data needed for your analysis prior exporting makes managing the dataset easier.

a. Select display parameters: In "Well configuration," select the wells you want to work with (Figure 2). On the top, select to display the parameter you would like to use and under the graph select to display the frequency. For cell growth, use capacitance (C) at a high frequency ( 32 or $64 \mathrm{kHz}$ ). For transepithelial resistance, select $Z$ at a low frequency (we use $500 \mathrm{~Hz})$.

b. Normalize data: This can be useful for comparing measurements from different days, or if the variability of the starting values makes comparisons difficult. In the "Time series" option, click "Normalize". Use the $0 \mathrm{~h}$ point for normalization. This will ensure that data collected on different days can be compared. Figures $3 \mathrm{~A}$ and $3 \mathrm{~B}$ show a measurement before and after normalization.

c. Combine parallel measurements: You have the option to combine all parallel measurements and export them as a single dataset, to limit the size of the exported dataset. For this in "Well Configuration" select the wells that represent parallel measurements. Click on Grp (group). This will calculate the average of your measurements and display them as one curve. Calculate the average for each condition (Figure 3C).

d. Make any other adjustment for exporting data: Adjust the graph to reflect the data you want to export: In "Well configuration" (bottom left), select the wells to export (either single measurements or the grouped value of the different conditions). Under the graph, adjust the $\mathrm{X}$-axis to display the required time frame (Figure 2). 


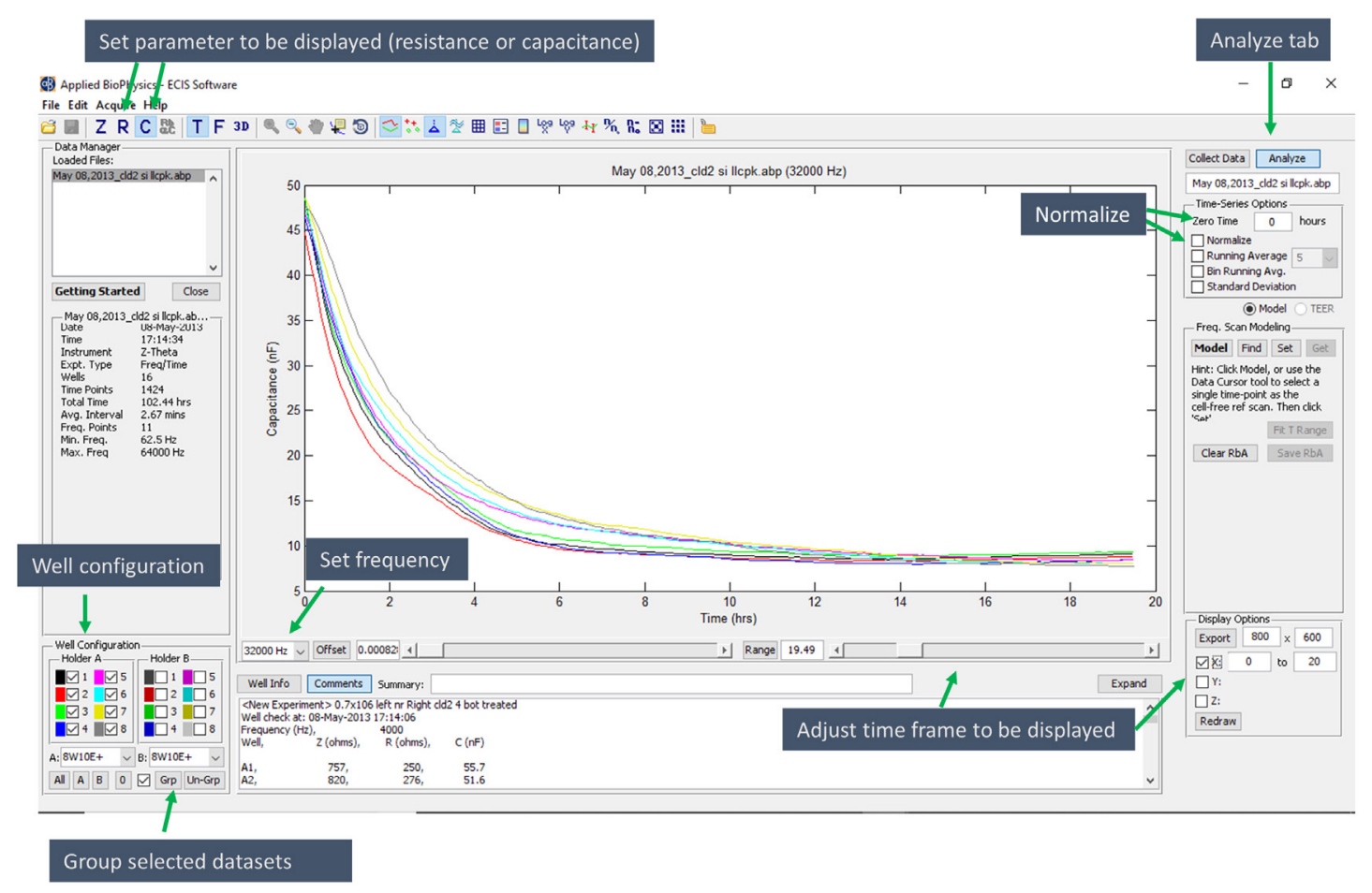

Figure 2. Screenshot from the ECIS software displaying results from a typical measurement described in the protocol. The cells were grown in an $8 \mathrm{~W} 10 \mathrm{E}+$ array, placed in Holder A. Conditions were as follows: 1-4 (left side): NR siRNA transfected; 5-8 (right side) Cldn-2 siRNA transfected. The capacitance displayed is set to $32 \mathrm{kHz}$. The various options in the software described in the protocol are marked with green arrows and blue text boxes indicate the various options. 
A

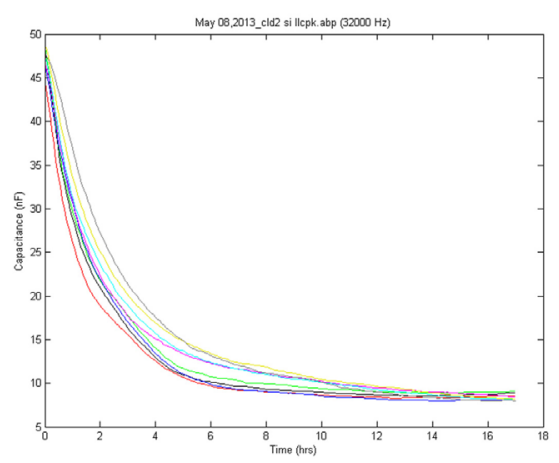

C

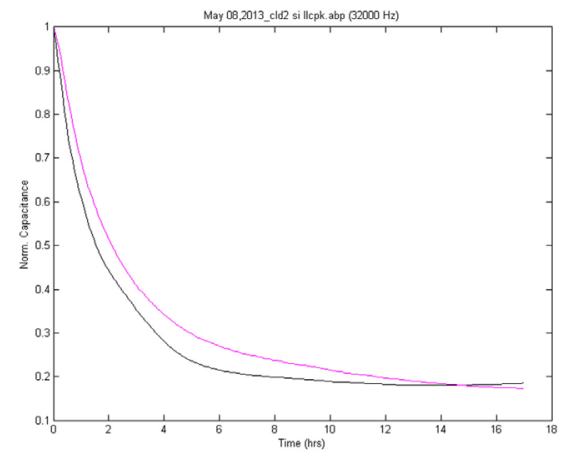

B

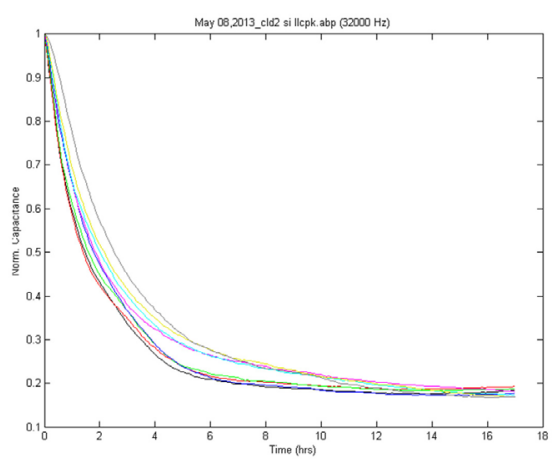

D

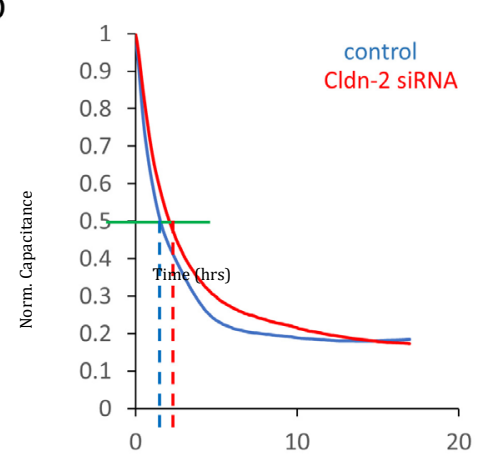

Figure 3. Data exported using the ECIS software with or without various processing. A-C. Exported graphs with various steps of analysis, as described in the protocol. A. All data are shown (graph from Figure 1). B. Data were normalized to the first point of the measurement. C. Data grouped according to the conditions. Black: NR siRNA; pink: Claudin-2 siRNA. D. Determination of half revovery time. Data were exported to Excel and graphed using a scatter graph with line option. Blue: NR siRNA; red: Claudin-2 siRNA. The green horizontal line indicates $0.5 \mathrm{C}$ value ( $\mathrm{Y}$ axis), and the blue and red dashed lines indicate the corresponding time ( $X$ axis value).

2. Exporting graph image and data

The graph can be exported as an image using the "File", "Export graph" command. Once the image to be exported opens, chose the save icon on top, and set the file name and format you wish to use. This image however will be of low quality with small fonts. Another option is to export the data from the graph and process using a graph software. For this open "File", select "Export data" and choose "Graph data". This mode of exporting will only save the data displayed on the graph in a *.csv format. You can also export all the data with the corresponding option in the "Export data" command. This will result in exporting all data collected at all frequencies.

3. Processing using a graphing software

Although the graph image can be exported, and the resulting picture has all the labels, these cannot be altered, and the graph and labels are of low quality. Therefore, we usually export the data to a graphing program for further processing and for generating publication quality graphs (Figure 3D). 


\section{Analyzing TER}

One mode of comparing barriers in two conditions is to compare $Z$ at a given timepoint after junctions developed. In Figure $1 \mathrm{~B}$ we graphed the $Z$ values from two measurements (those shown in Figure $1 \mathrm{~A}$ bottom graph and a similar series of experiment from a different day).

5. Analyzing cell growth

To compare data obtained in different experiments, we calculate for each measurement the time required for the cells to cover half the electrode (the time corresponding to $0.5 \mathrm{C}$ value). For this analysis we use the data from the capacitance (C) values at $32 \mathrm{kHz}$, normalized to the first measurement point (see step 2) (Figure 3D). The time values for all conditions are collected, and the mean \pm S.D. for each condition is calculated.

6. Statistics

The measurements must be performed at least in 3 different experiments. We use at least duplicates for every condition on every array. Direct comparison between arrays can pose a challenge, as the raw values show variability in different experiments. For example, TER data combined from two arrays obtained on different days shown on Figure 1B reveal that data from the same experiment cluster closer together, but the values differ from those measured on another array. While in both cases the claudin-2 siRNA transfected cells show elevated TER, unpaired $t$-test on the combined dataset shows that the change is not significant. Normalizing and expressing data as fold changes can solve this problem, as described for the $\mathrm{C}$ values. If that method is chosen, a non-parametric statistical test should be used.

\section{Notes}

1. Array type: There are various types of electrode arrays available, that differ in the number of electrodes. The more electrodes an array has, the more cells are being measured and averaged, resulting in reduced noise, but also smaller sensitivity. It is important to choose the appropriate electrode based on the experiment type. For a detailed description of each of the ECIS cultureware, users are referred to the Applied BioPhysics website: https://www.biophysics.com/cultureware.php.

For detecting cell growth and junction development, we used both $8 \mathrm{~W} 10 \mathrm{E}$ or $8 \mathrm{~W} 10 \mathrm{E}+$. The $10 \mathrm{E}+$ has more electrodes to collect information. Importantly, for a given series of experiment, always use the same array.

2. Electrode coating: Depending on the properties of the cell type used, collagen, fibronectin, laminin or other extracellular matrix proteins can be used to coat the electrode prior to seeding cells. Extracellular matrix proteins aid in cell adhesion and regulate a wide range of cell functions such as cell proliferation, differentiation, migration and survival. The type of protein used for coating might also affect proliferation and the development of the barrier, although this has to be systematically explored for specific cells. Therefore, it is important to standardize conditions of coating across experiments. We found that LLC-PK 1 cells do not require special coating, as 
they grow well on the gold electrodes.

3. Cell number and seeding: The inoculation technique is key for comparability and reproducibility between wells. Difference in proliferation can be masked by a high variability between wells. This usually results from inconsistency of seeding, and variation in cell number. For accurate measurements, cell numbers must be kept constant across the wells. Preparing an even cell suspension for seeding is key. Thoroughly trypsinize cells and mix suspension to eliminate clusters of cells. Accurate counting of the cell suspension to obtain comparable numbers when using cells from different conditions (e.g., transfection) is also important. Finally, prewarming of the array and the cell suspension to eliminate temperature difference between the well bottom and the suspension is also vital. For following cell growth, low cell number must be used to allow the cells to proliferate after adhering. If the cell number is too high, the cells will be confluent after adhering and no proliferation will occur. Cell adhesion and spreading is usually complete in the first $3 \mathrm{~h}$, and after this time frame the cells establish the confluent layer via proliferation.

4. Mycoplasma contamination of the cell lines should be routinely tested.

5. Transfection parameters: Transfection conditions vary depending on the cell dilines used and the target proteins, therefore, optimization of the conditions may be required. Knockdown efficiency of the target protein(s) should be confirmed by western blotting or qPCR in parallel. Changes in confluence in the transfected cells should be assessed using the capacitance measurement. The cells on the arrays can be visualized using a microscopy. In addition, effects on individual tight junction proteins are also informative and can be assessed by immunofluorescence.

6. Choice of frequency for capacitance and impedance measurement: When setting up the system, it is recommended to use the setting that collects data at all available frequencies. Due to differences in the resistance against the flow of current through the cells and between the cells, parameters measured at different $A C$ frequencies will reflect different aspects of cell properties. During analysis, the user can choose the frequency for the parameters of interest. Capacitance determined at high frequency reflects the coverage of the electrode, with minimal influence by other parameters, such as barrier function. The current recommendation and default in the software for analysis of capacitance values is $64 \mathrm{kHz}$. Of note, an earlier version of the software had $32 \mathrm{kHz}$ as the highest and default $\mathrm{C}$ value, and therefore we used this setting in our earlier studies. At this frequency, $\mathrm{C}$ also reflects electrode coverage. In contrast, resistance of the paracellular space (referred to as barrier function, generated by the tight junctions) is best reflected by low AC frequencies. Impedance $(Z)$ values at $500 \mathrm{~Hz}$ and below reflect dominantly resistance of the paracellular space. The software is also able to perform a mathematical modelling that allows further analysis and better distinction between resistance of the paracellular space and the space underneath the cells (cell-substrate attachment). For details the author is referred to Stolwijk et al. (2015). 


\section{$\underline{\text { Recipes }}$}

1. $10 \mathrm{mM} \mathrm{L-cysteine} \mathrm{solution} \mathrm{for} \mathrm{electrode} \mathrm{stabilization}$

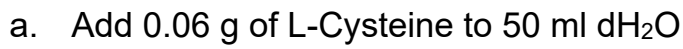

b. Filter to sterilize

c. Cysteine can easily oxidize when dissolved in water and therefore, this solution must be prepared fresh

\section{Acknowledgments}

Funding: Kidney Foundation of Canada; Canadian Institutes of Health Research (CIHR) grants PJT149058 and MOP-142409.

This protocol was derived from: Amoozadeh et al., 2015; Dan et al., 2019.

\section{Competing interests}

The authors declare that they have no financial or non-financial conflicts of interest.

\section{References}

1. Amoozadeh, Y., Anwer, S., Dan, Q., Venugopal, S., Shi, Y., Branchard, E., Liedtke, E., Ailenberg, M., Rotstein, O. D., Kapus, A. and Szászi, K. (2018). Cell confluence regulates claudin-2 expression: possible role for ZO-1 and Rac. Am J Physiol Cell Physiol 314(3): C366C378.

2. Amoozadeh, Y., Dan, Q., Anwer, S., Huang, H. H., Barbieri, V., Waheed, F., Maishan, M. and Szaszi, K. (2017). Tumor necrosis Factor- $\alpha$ Increases claudin-1, 4, and 7 expression in tubular cells: role in permeability changes. J Cell Physiol 232(8): 2210-2220.

3. Amoozadeh, Y., Dan, Q., Xiao, J., Waheed, F. and Szaszi, K. (2015). Tumor necrosis factor- $\alpha$ induces a biphasic change in claudin-2 expression in tubular epithelial cells: role in barrier functions. Am J Physiol Cell Physiol 309(1): C38-50.

4. Dan, Q., Shi, Y., Rabani, R., Venugopal, S., Xiao, J., Anwer, S., Ding, M., Speight, P., Pan, W., Alexander, R. T., Kapus, A. and Szászi, K. (2019). Claudin-2 suppresses GEF-H1, RHOA, and MRTF, thereby impacting proliferation and profibrotic phenotype of tubular cells. J Biol Chem 294(42): 15446-15465.

5. García, E., Pérez, P., Olmo, A., Díaz, R., Huertas, G. and Yúfera, A. (2019). Data-analytics modeling of electrical impedance measurements for cell culture monitoring. Sensors (Basel) 19(21): e4639.

6. Hagen, S. J. (2017). Non-canonical functions of claudin proteins: Beyond the regulation of cellcell adhesions. Tissue Barriers 5(2): e1327839. 
7. Kakiashvili, E., Dan, Q., Vandermeer, M., Zhang, Y., Waheed, F., Pham, M. and Szászi, K. (2011). The epidermal growth factor receptor mediates tumor necrosis factor- $\alpha$-induced activation of the ERK/GEF-H1/RhoA pathway in tubular epithelium. J Biol Chem 286(11): 92689279.

8. Láng, O., Köhidai, L. and Wegener, J. (2017). Label-free profiling of cell dynamics: A sequence of impedance-based assays to estimate tumor cell invasiveness in vitro. Exp Cell Res 359(1): 243-250.

9. McClatchey, A. I. and Yap, A. S. (2012). Contact inhibition (of proliferation) redux. Curr Opin Cell Biol 24(5): 685-694.

10. Stolwijk, J. A., Matrougui, K., Renken, C. W. and Trebak, M. (2015). Impedance analysis of GPCR-mediated changes in endothelial barrier function: overview and fundamental considerations for stable and reproducible measurements. Pflugers Arch 467(10): 2193-2218.

11. Szaszi, K., Vandermeer, M. and Amoozadeh, Y. (2012). Epithelial Wound Healing and the Effects of Cytokines Investigated by ECIS. Jiang, W. (Ed.). In: Electric Cell-Substrate Impedance Sensing and Cancer Metastasis. Cancer Metastasis - Biology and Treatment, vol 17. Springer, Dordrecht. 131-175.

12. Tsukita, S., Tanaka, H. and Tamura, A. (2019). The Claudins: From Tight Junctions to Biological Systems. Trends Biochem Sci 44(2): 141-152.

13. Venugopal, S., Anwer, S. and Szaszi, K. (2019). Claudin-2: Roles beyond Permeability Functions. Int J Mol Sci 20(22): 5655.

14. Wegener, J., Keese, C. R. and Giaever, I. (2000). Electric cell-substrate impedance sensing $(E C I S)$ as a noninvasive means to monitor the kinetics of cell spreading to artificial surfaces. Exp Cell Res 259(1): 158-166.

15. Zihni, C., Mills, C., Matter, K. and Balda, M. S. (2016). Tight junctions: from simple barriers to multifunctional molecular gates. Nat Rev Mol Cell Biol 17(9): 564-580. 